\title{
Ulrich Rüger
}

\section{Krankengeschichte und Lebensgeschichte}

Die biographische Dimension im Menschenbild der Medizin

$\because \because \because \because \because \because \because \because \because \because \because \because \because \because \because \because \because \because \because \because \because \because \because \because \because \because \because \because \because \because 9$

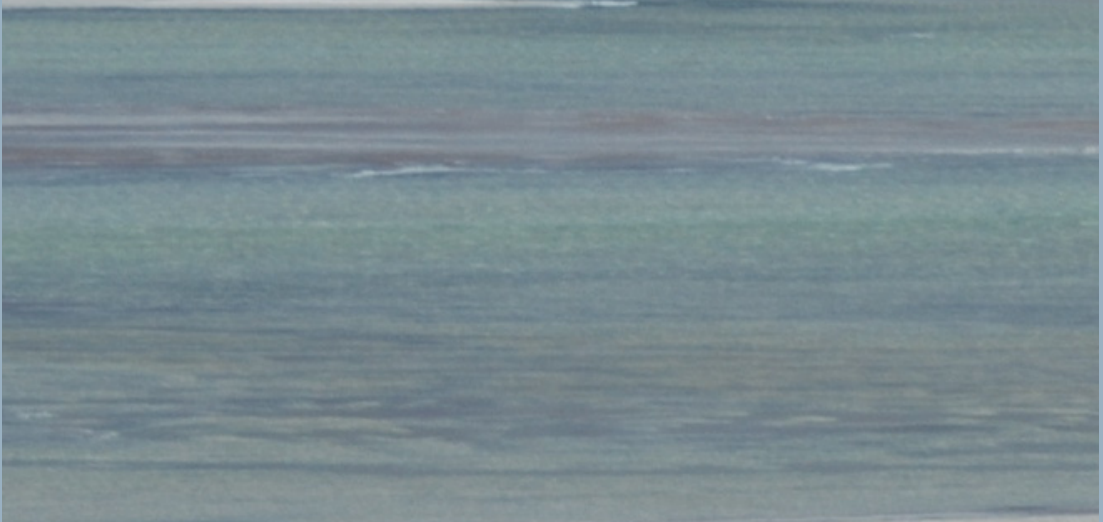

\section{Universitätsdrucke Göttingen}



Ulrich Rüger

Krankengeschichte und Lebensgeschichte

This work is licensed under the Creative Commons License 2.0 "by-nd", allowing you to download, distribute and print the document in a few copies for private or educational use, given that the document stays unchanged and the creator is mentioned. You are not allowed to sell copies of the free version.

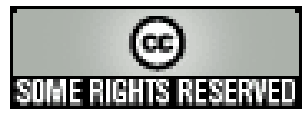


erschienen in der Reihe der Universitätsdrucke im Universitätsverlag Göttingen 2009 
Ulrich Rüger

Krankengeschichte und

Lebensgeschichte

Die biographische Dimension im Menschenbild der Medizin

Abschiedsvorlesung

am 15. Juni 2007

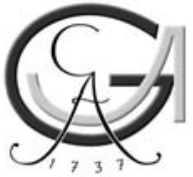

Universitätsverlag Göttingen 2009 


\section{Bibliographische Information der Deutschen Nationalbibliothek}

Die Deutsche Nationalbibliothek verzeichnet diese Publikation in der Deutschen Nationalbibliographie; detaillierte bibliographische Daten sind im Internet über $<$ http://dnb.ddb.de> abrufbar.

Dieses Buch ist auch als freie Onlineversion über die Homepage des Verlags sowie über den OPAC der Niedersächsischen Staats- und Universitätsbibliothek (http://www.sub.uni-goettingen.de) erreichbar und darf gelesen, heruntergeladen sowie als Privatkopie ausgedruckt werden. Es gelten die Lizenzbestimmungen der Onlineversion. Es ist nicht gestattet, Kopien oder gedruckte Fassungen der freien Onlineversion zu veräußern.

Satz und Layout: Ute Boldt

Umschlagabbildung: Salzsee in der Atacama-Wüste, Chile ( ${ }^{\odot}$ Gerhard Wörner)

(C) 2009 Universitätsverlag Göttingen

http:/ / univerlag.uni-goettingen.de

ISBN: 978-3-940344-79-3 
Nicht was wir gelebt haben, ist das Leben, sondern das, was wir erinnern und wie wir es erinnern, um davon zu erzäblen.

(Gabriel Garcia Márques, 2002) 



\section{Vorwort}

Das Thema Krankengeschichte und Lebensgeschichte steht nicht im Mittelpunkt der Forschung. Gleichwohl hat es unverändert große Bedeutung nicht nur für das von mir vertretene Gebiet der Psychosomatischen Medizin, sondern für die Medizin überhaupt, droht doch bei jeder schweren Erkrankung, ein bis dahin tragfähiges Lebensgleichgewicht verloren zu gehen. Wie in keinem anderen Beruf sind daher Ärztinnen und Ärzte mit den sehr unterschiedlichen biographischen Krisen ihrer Patienten konfrontiert und begleiten diese dabei, einen neuen Lebenskontext zu gewinnen. Diese biographische Dimension im Menschenbild der Medizin ist Gegenstand der nachfolgenden Ausführungen. Sie sind als Gedanken eines Menschen zu verstehen, der über 40 Jahre als Arzt tätig gewesen ist - darunter mehr als zwei Jahrzehnte als Hochschullehrer für Psychosomatische Medizin und Psychotherapie in Göttingen.

Für den Druck wurde der Text der Abschiedsvorlesung nur leicht überarbeitet. Ihr Aufbau blieb unverändert. Weiterführende Aspekte werden in Fußnoten berücksichtigt.

\section{Ulrich Rüger}





\section{Historische Anmerkungen}

In seiner Epikrise zum Fall der Elisabeth v. R. schrieb Sigmund Freud 1895: „Ich bin nicht immer Psychotherapeut gewesen, sondern wie andere Neuropathologen erzogen worden ... und es berührt mich selbst noch eigentümlich, dass die Krankengeschichten, die ich schreibe, wie Novellen $\mathrm{zu}$ lesen sind, und dass sie sozusagen des ernsten Gepräges der Wissenschaftlichkeit entbehren“. (GW I, S. 227).

Sigmund Freud war ein Kind seiner Zeit, der beginnenden naturwissenschaftlichen Ära der Medizin. Und Freud war Schüler des Physiologen Ernst von Brücke in Wien gewesen und fühlte sich zeitlebens dessen Ideal von Wissenschaft und intellektueller Unbestechlichkeit verpflichtet. ${ }^{1}$ Dabei gilt Brücke, gemeinsam mit dem Berliner Physiologen du Bois-Reymond, als einer der wesentlichen Begründer einer naturwissenschaftlich orientierten Medizin. Letzterem wird folgendes Zitat zugeschrieben: „Brücke und ich, wir haben uns verschworen, die Wahrheit geltend zu machen, dass im Organismus keine anderen Kräfte wirksam sind, als die gemeinen physikalisch-chemischen". ${ }^{2}$

Was viele nicht sehen, dieses Zitat war nicht nur in die Zukunft gerichtet. Vielmehr grenzten sich Brücke und du Bois-Reymond hiermit ausdrücklich von ihrem gemeinsamen, langjährigen Lehrer, dem Berliner Physiologen Johannes Peter Müller ab. Dieser war seinerzeit als Vertreter des Vitalismus einer der letzten Repräsentanten der romantischen Medizin der ersten

\footnotetext{
$1 \mathrm{Vgl}$. Ernest Jones, 1960, S. $60 \mathrm{f}$

2 vgl. Rüger 1996, Hoffmann et al. 1999
} 
Hälfte des 19. Jahrhunderts. Als verhinderter Historiker möchte ich aber nicht ausufern. Was Sie sehen, ist aber: Die Pioniere neuer wissenschaftlicher Entwicklungen haben diese auch häufig nur in einer (oft überbetonten) Gegenposition zu ihrer Vorgeneration in Gang setzen können - ein häufiges Phänomen in der Genealogie unserer Wissenschaftsgeschichte.

Zurück zu Sigmund Freud: Seine eben zitierten Anmerkungen in den „Studien über Hysterie“ entbehren nicht eines gewissen defensiven Charakters - vorgetragen in der Freud eigentümlichen Selbstironie. Dabei hätte er seinerzeit durchaus einen wortmächtigen Verbündeten finden können. Den hat er aber offensichtlich nicht wahrgenommen:

Es handelt sich um den Philosophen Wilhelm Windelband. Dieser hatte zunächst auch Medizin und Naturwissenschaften studiert, sich dann aber der Philosophie zugewandt und mit seiner berühmten Straßburger Rektoratsrede 1894 das Spannungsfeld zwischen den idiographischen (d. h. einzelfallorientierten, biographischen) Forschungsansätzen und nomothetischen (d. h. nach übergreifenden Gesetzmäßigkeiten suchenden) Forschungsansätzen herausgearbeitet - übrigens in diesem Punkt ein ideengeschichtlicher Vorläufer des Heidelberger Psychiaters und Philosophen Karl Jaspers, der als nachhaltiger Psychoanalyse-Kritiker dieser wiederum vorwarf, die Grenzen zwischen biographischem Verstehen und kausalem Erklären einer Erkrankung zu verwischen. ${ }^{3}$

Lassen Sie mich dieses Problem an einer Krankengeschichte verdeutlichen. Vielleicht werden Sie den Patienten im Verlauf des Berichtes erkennen:

3 vgl. Jaspers 1965 


\section{Die Krankengeschichte des jungen G.}

Ein junger Mann verlässt seine Vaterstadt Frankfurt, um in Leipzig Rechtswissenschaften zu studieren. Hätte er seinen Neigungen folgen dürfen, so wäre er nach Göttingen gegangen, um sich dort dem Studium der Altertumswissenschaften zu widmen. Nach den Wünschen des Vaters jedoch sollte er wie dieser an dessen alter Universität Jura studieren, um später die Laufbabn eines böheren Verwaltungsjuristen einzuschlagen - das ursprüngliche Ziel des Vaters, dass dieser seinerzeit verfehlt hatte.

In seiner neuen Leipziger Umgebung füblte sich der junge Mann einsam und von Heimweh geplagt. Erste Liebessehnsüchte zu der jungen Tochter seiner Wirtsleute blieben unerfüllt. Zum Studieren kam er kaum. Er zog sich zunehmend zurück und entwickelte eine Vielzabl von Krankheitssymptomen: Verstopfungen, bäufige Infekte, eine Geschwulst am Halse machte ibm zu schaffen, und er litt unter häufigen Zahnschmerzen, beständigem Husten, Arbeitsstörungen und bypochondrischen Befürchtungen. Verschiedene mediainische Behandlungsmaßnahmen wurden unternommen und unterschiedliche Expertisen über seinen Krankheitszustand abgegeben. Sie reichten von einer vermuteten syphilitischen Infektion, dem Verdacht einer Tuberkulose bis bin zu einer psychischen Erkrankung. Der Patient selbst hatte das Gefübl, dass ibm „das Gebirn verdüstert und die Eingeweide paralysiert" seien. Während dieser Zeit schwankte er zwischen Ausgelassenheit und tiefer Verstimmung, die in Selbstmordphantasien mündeten.

Am Ende seiner dreijährigen Leipziger Zeit geriet er wiederum in eine schwere Krise, die schließlich einen physischen Zusammenbruch auslöste. Er erlitt einen Blutstury, schwankte mehrere Tage zwischen Leben und Tod und kehrte gleichsam als "Schiffbrüchiger" in seine Vaterstadt zurück. Fast eineinhalb Jahre dauerte es noch, bis er sich wieder erholt hatte und sich seine Befürchtungen, die Schwindsucht zu haben, verloren,.

Schließlich konnte er Ostern 1770 zum zweiten Mal sein Vaterhaus verlassen, um das unterbrochene Studium in Straßburg zu beenden. Die dortigen eineinbalb Jabre erlebte er, wie keine andere Periode seines Lebens, als Neubeginn und widmete sich neben der zwangsläufigen Beschäftigung mit der Juristerei auch der Philosophie, Theologie, den Naturwissenschaften und der Medizin. 
Sie werden inzwischen bemerkt haben, um wen es sich handelt: Es ist die Geschichte des jungen Studenten Johann Wolfgang Goethe 4 und bei aller Vorsicht dürfen wir folgendes sicherlich feststellen: Die sehr zwiespältige Akzeptanz des väterlichen Lebensentwurfes - der Sohn sollte für den Vater nicht Vollendetes in dessen Entwurf erreichen! - ging der Erkrankung voraus. Die Krise nach dem dreijährigen Jurastudium - „zu der ihn keine innere Richtung drängte“ - war zugleich Chance für die Auseinandersetzung mit seinem bisherigen, vom Vater bestimmten Lebensentwurf. Bei dieser Auseinandersetzung war ihm sein behandelnder Arzt Johann Friedrich Metz Freund und väterliches Vorbild zugleich. Er eröffnete ihm das Interesse für neue Perspektiven: Für die historische Gestalt des Paracelsus sowie für Medizin und Naturphilosophie.

Mit der Aufnahme des Studiums in Straßburg sprengte der junge Goethe den väterlichen Horizont und Entwurf - auch wenn er dann bereits sechs Jahre später bis zu seinem Lebensende als Verwaltungsjurist in Weimar sein Brot verdiente und damit, so können wir es sehen, die väterliche Linie wieder aufnahm.

Er tat dies aber in einer völlig anderen Form! Er hatte seinen eigenen Lebensentwurf gefunden - auch wenn sein späteres Leben nicht frei von Krisen blieb.

Diese Krankengeschichte zeigt uns aber auch: Es ist müßig zu erörtern, ob das ungeliebte Studium Goethe krank gemacht hat oder seine körperliche Erkrankung Krise und Chance zu einer Neuorientierung wurden - bei der ihm dann sein Arzt behilflich war. Das ist letztlich Spekulation. Und hier hat sicherlich Sören Kierkegaard mit seiner Feststellung recht: Das Leben wird vorwärts gelebt und rückwärts verstanden. ${ }^{5}$ Das heißt, wir geben im Nachhinein Entwicklungen einen Sinn und setzen dann oft Sinn und Ursache

4 vgl. z. B. Boerner, 1964

5 zitiert nach Esch, 1994 
gleich! Aber dass wir das tun können, hält uns in der Welt und trägt zu unserem inneren Gleichgewicht bei. Und deshalb haben zu allen Zeiten Menschen nach Gesetzmäßigkeiten im Ablauf des Lebens gesucht - das gilt insbesondere für die Medizin. Soweit wir hier schriftliche Aufzeichnungen vorliegen haben, ging es nie nur um Krankheit und Gesundheit, sondern immer auch um das Bild vom Menschen und um Vorstellungen über biographische Gesetzmäßigkeiten im Ablauf unseres Lebens.

\section{Psychosomatische Medizin - Biographische Medizin}

Von den 20er Jahren des letzten Jahrhunderts an war es schließlich Viktor von Weizsäcker, der die Biographische Methode als Zugang zur subjektiven Seite des Patienten in die Medizin einführte und zum Begründer der Anthropologischen Medizin wurde: „Wenn man aber die Einbettung organischer Erkrankungen in die äußere und innere Lebensgeschichte erkundet, so ist man erstaunt, wie oft Krankheit auf dem Gipfel einer dramatischen Zuspitzung auftritt, wie oft sie eine Katastrophe aufhält oder besiegelt, wie regelmäßig sie dem biographischen Verlauf eine neue Wendung gibt““.6 Damit ist für mein Thema eigentlich alles schon gesagt! ${ }^{7}$

Übrigens hat selbst Hans Selye, der als Internist und Physiologe Begründer der modernen Stressforschung wurde und damit eine biologische Wurzel der psychosomatischen Medizin mitbegründet hat, in der zweiten Hälfte seines wissenschaftlichen Lebens biographischen Faktoren durchaus einen Stellenwert beigemessen: Er musste konstatieren, dass das subjektive Erleben von objektiven Stressoren die nachfolgende Stressreaktion des Organismus wesentlich mitbestimmt (1955).

6 1955, gesamte Schriften Band VII, S. 380

7 Ausführliche Würdigung Viktor von Weizsäckers als „Vater der Psychosomatischen Medizin“ durch S. O. Hoffmann, 2006. 
Rüger: Krankengeschichte und Lebensgeschichte

Alexander Mitscherlich, damals noch ganz in der Tradition von Viktor von Weizsäckers stehend, definierte schließlich die Psychosomatische Medizin als „Biographische Medizin“. Bereits 1954 charakterisiert Mitscherlich allerdings von Weizsäckers Auffassung als „naive Biographik““. 8,9

Annemarie Dührssen war es dann schließlich, die mit ihrem in den 1960iger Jahren entwickelten Konzept der biographischen Anamnese nachhaltigen Einfluss auf das biographische Denken genommen und das Verständnis für die Lebensschicksale von Patienten sehr gefördert hat. So steht seit der Einführung der Psychotherapie in die kassenärztliche Versorgung 196710 die biographische Anamnese auch am Beginn einer jeden psychotherapeutischen Behandlung.

8 vgl. Thomae 1952, S. 175, Mitscherlich 1954; Dehli 2007, S. 213, Fußnote 112).

9 Mit seiner Hinwendung zur Psychoanalyse war nach Dehli (2007) nun nicht mehr „eine umfassende medizinische Lehre vom Menschen, sondern die psychoanalytische Frage nach Triebschicksalen .... Mitscherlichs primäres Interesse.“ (S. 184) Mit diesem Diktum Alexander Mitscherlichs und seiner Abwendung von der anthropologischen Medizin Viktor von Weizsäckers beginnt möglicherweise bereits damals in der Psychoanalyse das Interesse an Biographien zurückzugehen. So konstatiert Rudolf bei einem Vergleich typischer Weiterbildungsanamnesen aus den 60-iger und aus den 90-iger Jahren eine „Verringerung der Aufmerksamkeit für die biographische und aktuelle soziale Lebenswirklichkeit des Patienten und ... eine Zunahme der Beschreibung interpersonellem Geschehens zwischen Patient und Therapeut, ferner ... eine deutlich vermehrte Bereitschaft, theoriegeleitete Interpretationen $\mathrm{zu}$ formulieren“ (S. 29) - mit der Neigung Lebensdaten lediglich als Beweismittel theoretischer Interpretationen heranzuziehen (S. 30). Rüger et al. (1996) stellen bei einer entsprechenden Untersuchung fest, dass die Kenntnisse der Therapeuten über die Biographien ihrer Patienten sehr durch theoretische Vorannahmen selektiert werden. Das Interesse an Krankengeschichte und Lebensgeschichte tritt zurück zugunsten einer Zunahme theoriegeleiteter Interpretationen (Rudolf 1993). Cui bono?

Mit der Vernachlässigung des biographischen Kontextes ist auch in der Psychotherapie-Forschung eine zunehmende „dekontextualisierte“ Sicht des therapeutischen Handelns festzustellen. Dies wird inzwischen auch von maßgeblichen Vertretern der evaluativen Psychotherapie-Forschung beklagt (Orlinsky 2008).

10 vgl. Rüger 2007. 
Bei aller Betonung eines einzelfallorientierten idiographischen Ansatzes steht gleichwohl die Suche nach generalisierbaren Gesetzmäßigkeiten im Ablauf biographischer Entwicklungen - im Sinne eines nomothetischen Ansatzes! - ebenfalls auf der Agenda der Psychosomatischen Medizin und Psychotherapie. Damit nicht der Eindruck entsteht, die Psychosomatische Medizin sei nur für die subjektive Seite des Menschenlebens zuständig, folgt zunächst ein kurzer Exkurs zu den biographischen Modellen von Krankheit und Gesundheit und dem Stand ihrer empirischen Überprüfung. 



\section{Biographische Modelle von Krankheit und Gesundheit und ihre empirische Überprüfung}

Die Frage ist hier immer: Wie bin ich geworden, was macht mich krank, was hält mich gesund? Das ist aber nicht eine Frage der Psychosomatischen Medizin allein, sondern der Medizin insgesamt. Alle empirischen Befunde, auf die ich heute im Einzelnen nicht eingehen kann, sprechen dafür, dass wir von Umwelt und genetischer Ausstattung in gleicher Weise geprägt sind und dass beide in jeder Lebensphase wesentlich mit dafür verantwortlich sind, ob wir gesund bleiben oder krank werden und - wenn wir krank geworden sind - ob wir die Krankheit gut verarbeiten können und wieder gesunden oder chronisch krank bleiben. Früher wurden diese Zusammenhänge eher additiv gesehen. Inzwischen wissen wir, dass von den vorhandenen Genen einer Zelle nur ein kleiner Teil zur Synthese von Protein abgelesen wird. $\mathrm{Ob}$ es dann zu einer Genexpression kommt, wird maßgeblich von Umwelteinflüssen, von Umweltbelastungen mitbestimmt, d. h.: Die Verbindung zwischen genetischer Disposition und Umwelteinflüssen ist sehr viel enger als früher angenommen. 


\section{Abb. 1}

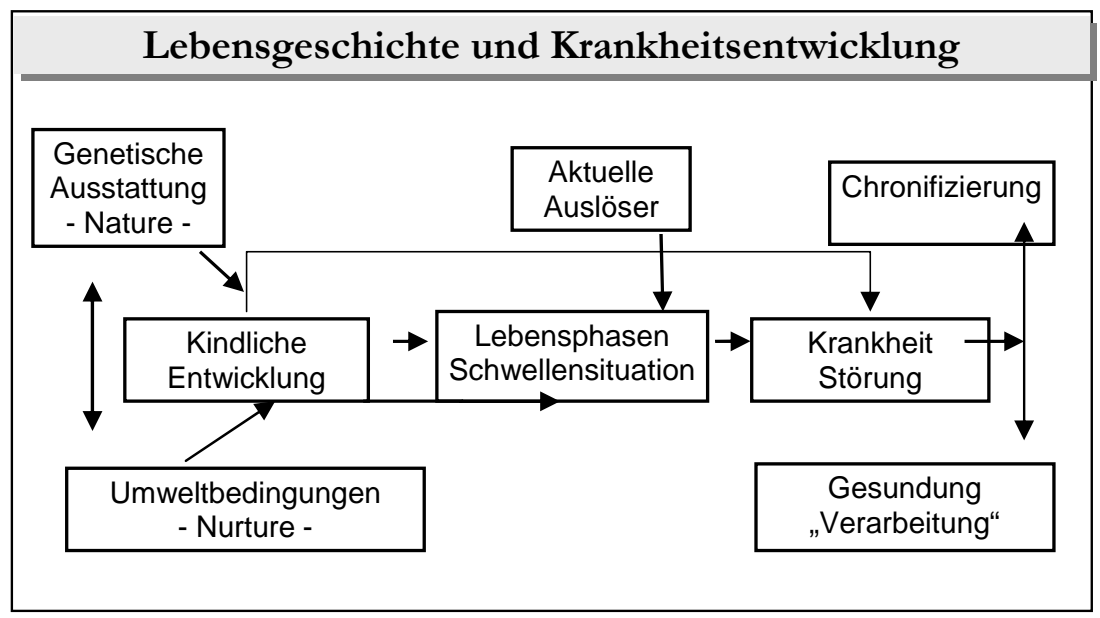

Neben diesem komplexen Zusammenspiel zwischen genetischen und Umwelt-Faktoren gilt heute eine Interaktion zwischen pathogenen und protektiven bzw. salutogenen Faktoren in der Lebensentwicklung als selbstverständlich. Erlauben Sie in diesem Zusammenhang einige schon als historisch zu nennende Langzeitstudien in Erinnerung zu rufen: 1977 wurden von Vaillant in der Monographie „Adaption to life“ die Ergebnisse einer prospektiven Langzeitstudie mitgeteilt, die bereits 40 Jahre vorher 1937 begonnen worden war und kontinuierlich vom NIMH gefördert wurde. Das interessante an dieser Studie ist nicht nur die große Zahl an pathogenetischen Faktoren, die isoliert werden konnte, sondern auch sogenannte protektive (schützende) Faktoren in einer biographischen Entwicklung. Und besonders bedeutsam scheint mir der Befund, dass $60 \%$ der pathogenetischen Faktoren, die bei späteren Patienten festzustellen waren - also Risikofaktoren - auch bei lebenslänglich Gesunden anzutref- 
fen waren. Daraus wurde sicherlich zu Recht geschlossen, dass protektive und salutogene Faktoren für den Ausgang einer Lebensentwicklung in gleicher Weise wichtig sind und die eigentliche Bedeutung in deren dynamischem Zusammenspiel zu sehen ist.

$\mathrm{Zu}$ erwähnen ist die sehr gute Studie an Risiko-Kleinkindern aus dem Zentralinstitut für Seelische Gesundheit in Mannheim aus der Arbeitsgruppe des Kinderpsychiaters Schmidt. ${ }^{11}$ Hier wurde der Einfluss der Mutter-Kind-Interaktion auf die kognitive und sozial-emotionale Entwicklung einer Risikopopulation von Neugeborenen untersucht. Es konnte nachgewiesen werden, dass die Qualität der frühen Mutter-Kind-Beziehung einen maßgeblichen Einfluss auf eine günstige bzw. ungünstige Entwicklung dieser Kinder mit „neuropsychiatrischen Störungen“ hatte.

Dann darf an dieser Stelle die Arbeitsgruppe um Heinz Schepank, ebenfalls aus dem Zentralinstitut für Seelische Gesundheit in Mannheim, nicht unerwähnt bleiben: Unter anderem gefördert durch einen Sonderforschungsbereich (SFB 116) wurden hier die Determinanten von seelischer Gesundheit und Krankheit bei einer unausgelesenen Großstadtpopulation untersucht. ${ }^{12}$

Aus derselben Arbeitsgruppe ist dann schließlich Tress der Frage nach dem „Rätsel der seelischen Gesundheit“ (1986) nachgegangen. Er hat dabei wesentliche protektive Faktoren identifiziert, die trotz schwerer Frühbelastung letztlich einen Menschen lebenslänglich gesund bleiben lassen.

In die gleiche Richtung gehen die Untersuchungen von Antonowsky (1987), der bei Holocaust-Überlebenden deren Gesundheitszustand und die Art ihrer Lebensbewältigung überprüft hat und zu sehr beeindruckenden Ergebnissen gekommen ist - Grundlage und Anstoß des vom Autor entwickelte Salutogenese-Konzept. ${ }^{13}$ Etwa in der selben Zeit wurden von Emmy Werner (1989) Ergebnisse einer prospektiven Langzeituntersuchung

11 Vgl. Esser et al. 1993

12 Vgl. Schepank et al. 2000, Franz et al. 2000

13 Vgl. zu dieser Thematik auch die Langzeit-Katamnese an Holocaust-Überlebenden von Freyberger und Freyberger, 2007 
publiziert, in der die Autorin eine Hochrisiko-Population von Kindern auf Hawaii untersucht hat - mit dem Ergebnis, dass für den Ausgang der späteren Lebensentwicklung nicht so sehr die belastenden sondern die protektiven Faktoren ausschlaggebend sind.

Ich habe hier nur beispielhaft einige wenige, mich besonders beeindruckende Studien aufgeführt. Alle haben seinerzeit zu einem konzeptuellen Umdenken im Hinblick auf die pathogenetischen Bedingungen für Krankheit und Gesundheit geführt.

Die folgende Übersicht fasst noch einmal unser gegenwärtiges Wissen im Hinblick auf die maßgeblichen Risikofaktoren und protektiven Faktoren unserer biographischen Entwicklung zusammen. 
Abb. 2

\section{Risikofaktoren und protektive Faktoren bei psychischen / körperlichen Erkrankungen}

\section{Risikofaktoren (u. a.)}

- Niedriger sozioökonomischer Status

- Arbeitslosigkeit der Eltern

- Eltern kriminell/dissozial

- Mütterliche Berufstätigkeit im 1. Lebensjahr

- Unsichere Bindung vom 12. bis 18. Monat

- Psychische bzw. schwere körperliche Erkrankung der Eltern

- Chronisch kranke Geschwister

- Alleinerziehende Mutter

- Längere Trennung der Eltern vor dem 7. Lebensjahr

- Chronische Konflikte bei Trennung der Eltern

- Missbrauch

- Schlechte Kontakte zu Gleichaltrigen

- Abstand zu nächst. Geschw. < 18 Mon.

\section{Protektive Faktoren (u. a.)}

- Dauerhafte gute Beziehung zu mindestens einer Bezugsperson in der Kindheit

- Sicheres Bindungsverhalten

- Großfamilie, kompensatorische Beziehungen

- Entlastung der Mutter (wenn allein erziehend)

- Gutes Ersatzmilieu nach Mutterverlust

- Überdurchschnittliche Intelligenz

- Robustes, aktives, kontaktfreudiges Temperament

- Soziale Förderung (in Schulzeit / Pubertät) 
Es handelt sich hier um statistische Zusammenhänge zwischen möglichen Risikofaktoren und einer Reihe späterer psychischer, aber auch körperlicher Erkrankungen. Diese statistisch relevanten Zusammenhänge müssen aber im Einzelfall nicht gelten. Dort wirksame pathogene und protektive Faktoren können andere sein. Trotzdem ist die Suche nach generalisierbaren Gesetzmäßigkeiten im Sinne eines nomotetischen Vorgehens auch von großer praktischer Bedeutung, z. B. für Fragen der Prävention und Rehabilitation. Und die Suche nach solchen generalisierbaren Gesetzmäßigkeiten ist zwangsläufig reduktionistisch - man muss sich dessen nur bewusst bleiben.

Dem einzelnen Menschen aber werden wir nur gerecht, wenn wir uns in seine Lebensgeschichte vertiefen - ohne sofort „seelisches Leben in leicht operationalisierbare Fragmente aufzulösen“ oder „mit falschen Bruchstücken zu beginnen“, wie einer der Väter der BiographieForschung, Hans Thomae bereits 1952 gemahnt hat. Ansonsten liefen wir Gefahr, dass jeweils „,individuelle Gesetz“ (Georg Simmel 1918) eines Lebens zu übersehen.

„Biographik“ in einem solchen Sinne besagt, „dass wir es in einem menschlichen Leben nicht mit einer Abfolge beliebiger Vorkommnisse, also auch nicht mit einer fortlaufenden Kette objektivierbarer , life events' zu tun haben" ${ }^{14}$, sondern mit einer Abfolge von Geschehnissen. ${ }^{15}$ Und deren kontextueller Bezug geht in Lebenskrisen - also auch bei schwerer Krankheit - verloren.

Das klingt für Mediziner etwas theoretisch; und wie Walter Bräutigam (1989) seinerzeit einmal festgestellt hat, verträgt die handlungsorientierte klinische Medizin nur ein bestimmtes Maß an Theorie. Das nachfolgende Fallbei-

14 Vgl. Lang 2000, S. 42

15 Vgl. Blankenburg 1981 
spiel soll Ihnen plastisch das Zusammenspiel von Krankengeschichte und Lebensgeschichte vor Augen führen.

\section{Krankengeschichte der Patientin B.}

Die seinerzeit 79-jährige Patientin wurde mir auf Zuweisung eines unserer operativen Kollegen hier im Klinikum vorgestellt. Mein Bericht stützt sich auf die fünf Termine, zu denen die alte Dame zu mir kam, auf einige Telefonate mit der nachbehandelnden Hausärztin in einem weiter entfernten Ort und auf eine Telefon-Katamnese bei dieser Kollegin sowie Auskünfte über den weiteren Verlauf der körperlichen Erkrankung der Patientin.

Es handelte sich um eine trotz ihrer Depressivität vital und deutlich jünger erscheinende Patientin, die von Tränen unterbrochen sehr bereitwillig ihre Vorgeschichte schilderte: Bis vor zwei Jahren sei sie immer eine strahlende und aktive Frau gewesen. Nichts hätte sie umwerfen können. Dann sei alles für sie zusammengebrochen. Ihre körperliche Erkrankung ein metastasierendes Tonsillenkarzinom - war bereits vor vier Jahren diagnostiziert und in mehrfachen Operationen behandelt worden. Der psychische Zusammenbruch erfolgte also nicht in unmittelbarem Zusammenhang mit der Diagnose der Krebserkrankung.

Ihr Professor - so äußerte sie sich - würde sie nur für ein „belämmertes Familienleben“ am Leben erhalten, sie wolle nicht mehr leben, habe sich schon einen Strick besorgt. „Sie nehmen sowieso umsonst Ihre kostbare Zeit für mich in Anspruch!“

Ihr behandelnder Arzt hatte mir die Patientin bereits telefonisch angekündigt, war über die aktuelle Lebenssituation der Patientin sehr gut informiert und äußerte mir gegenüber nur, dass er die Patientin so wie sie sich jetzt bei ihm vorstelle, aus früheren Terminen nicht wiedererkenne. Sie sei psychisch völlig verändert. 
Die Patientin war deutlich depressiv herabgestimmt, zeigte massive Einschlaf- und Durchschlafstörungen, der Antrieb war eher etwas gesteigert, was die Suizidgefahr vergrößerte. Sie zeigte sehr starke Schuldgefühle gegenüber dem ein halbes Jahr zuvor im Rahmen eines demenziellen Prozesses verstorbenen Ehemann. Dieser hatte sich wegen einer vermeintlichen außerehelichen Beziehung der Patientin mit dieser bis zu seinem Tod nicht mehr versöhnt. Dabei dürfte es sich auf Seiten des Ehemannes um eine krankheitsbedingte Fehlerinnerung und Fehldeutung eines harmlosen Telefonats eines früheren Bekannten mit der Patientin gehandelt haben.

Ein Jahr später hatte die Patientin dann ihrem Enkelsohn - unter Überspringung des aus ihrer Sicht missratenen Sohnes - ihren Anteil an einem großen Geschäftshaus überschrieben. Er - der Enkel - habe sich bei ihr „eingeschmeichelt“ und sei nach den entsprechenden Unterschriften dann verschwunden. Jetzt werde auf sein Betreiben hin das Haus verkauft, und sie habe dort nicht einmal Wohnrecht! Sie stehe vor den Trümmern eines belämmerten Familienlebens. Ihr Leben habe keinen Sinn mehr.

Die Patientin wirkte nicht eigentlich wütend auf den Enkel oder die anderen beteiligten Familienmitglieder, sondern eher zutiefst beschämt, dass sie sich von dem jungen Mann durch vielerlei Schmeicheleien hatte einwickeln lassen.

In der dann folgenden Woche - schon etwas stabilisiert und nicht mehr akut suizidal - konnte die Patientin mir die sehr schwierige Beziehung zu ihrem Sohn (Heiratsanlass ihrer nur sehr kurz dauernden ersten Ehe 1935/36) schildern; und sie kam dann schließlich auch darauf, wie sie gehofft hatte, den Bruch mit ihrem Sohn durch eine bessere Beziehung zu dem Enkel wieder rückgängig machen zu können. Wir kamen dann auf ihre Empfänglichkeit für Schmeicheleien zu sprechen, die sich durch ihr ganzes Leben hindurchgezogen hatte - nicht nur in der jüngsten Vergangenheit! Sie war immer eine Attraktive, Große, Blonde, Blauäugige gewe- 
sen, der es seinerzeit sehr geschmeichelt hatte, aus vielen Bewerberinnen für eine Tätigkeit als Bibliothekarin bei einem sehr hochrangigen Naziführer ausgesucht worden zu sein.

31-jährig hatte sie ihren zweiten Ehemann, einen erfolgreichen Geschäftsmann, geheiratet. „Ich war aber die Stärkere!“ - „Ich wollte nicht unverheiratet bleiben!“ „Ich war sein Augenstern!“ So wie dereinst bei ihrem Vater! Mit ihrem Mann hatte sie dann bis zu dessen demenzieller Erkrankung eine gute Ehe geführt.

Nach den heutigen diagnostischen Gepflogenheiten würde man bei der Patientin eine temporäre komorbide schwere depressive Episode diagnostizieren, die nach kurzer medikamentöser und psychotherapeutischer Intervention remittierte. Wie blass und nichtssagend ist aber diese Diagnose einer komorbiden Störung gegenüber einem biographischen Verständnis für eine Lebenskrise!

Diese Krise entwickelte sich bei der Patientin nicht nach der Krebserkrankung und den damit verbundenen ersten Behandlungsmaßnahmen. Vielmehr war hier eine mehrstufige Entwicklung festzustellen. Wie häufig bei schweren Erkrankungen war zu unterscheiden zwischen:

- Lebensveränderungen als Folge einer schweren Erkrankung (z. B. auch notwendigen Behandlungsmaßnahmen)

- Lebensveränderungen in zeitlicher Koinzidenz mit einer Erkrankung (hier z. B. demenzielle Entwicklung des Ehemannes)

- Lebensentscheidungen wegen einer schweren Erkrankung (mit ggf. familiären und sozialen Folgeerscheinungen)

Im Rahmen dieser Entwicklung war es zu der geschilderten Situation gekommen. Und dies ist es, was ich Ihnen, insbesondere auch den Studierenden, d. h. den angehenden Ärzten, vermitteln möchte: Es ist nicht immer 
die Krankheit selbst, die in die Krise führt, sondern der damit verbundene Verlust von Kohärenzregeln ${ }^{16}$, mit denen wir bisher das Leben bewältigt haben und die bis dahin unserem Handlungs- und Erfahrungsraum Grenzen und Richtung gegeben haben. Man kann auch von einer „kontextuellen Krise" sprechen, in der der Zusammenhang des bisherigen Lebens bedroht ist oder vollends verloren geht.

In solchen Krisen setzt eine oft aufwändige Dekonstruktion unseres Bildes von unserem bisherigen Leben ein, gefolgt von einer oft sehr mühseligen Rekonstruktion, an deren Ende meist ein verändertes Bild von der eigenen Biographie steht - Grundlage für ein verändertes Leben.

Triebfeder dabei ist das Bedürfnis des Menschen nach KontingenzErfahrung mit deren Hilfe „der Mensch mit der Zerrissenheit seines Daseins, Ohnmacht und Ausgeliefertsein an Zufälle fertig wird“ “. ${ }^{17}$ Von den inneren Prozessen, die durch solche Lebenskrisen angestoßen werden und jede schwere Erkrankung kann eine solche Krise hervorrufen - sollten Ärztinnen und Ärzte Kenntnis haben und diese im Umgang mit ihren Patienten berücksichtigen.

Ich komme noch einmal zurück zu unserer Patientin: Wie ich nach einer Sechs-Monats-Katamnese bei der Hausärztin erfahren konnte, hatte sich die Patientin rasch stabilisiert, die antidepressive Medikation konnte bereits nach drei Monaten abgesetzt werden.

Den späten Versuch, über den Enkel erstmals in ihrem Leben eine intakte Familie zu bekommen - eine im Kontext ihres bisherigen Lebens sicherlich eher fragwürdige Lösung -, hatte sie offensichtlich aufgeben können und gewann zunehmend - so wie die Hausärztin berichtete - Kontakte zu ihrer eigenen Altersgruppe.

86-jährig verstarb sie an den Spätfolgen ihrer onkologischen Erkrankung.

16 Vgl. H. Bude 1999, S.252

17 Vgl. Boehme 1990 
Bei der Durchsicht ihrer Krankengeschichte fand sich als letztes Blatt ein Schreiben an ihren Arzt, der sie über lange Jahre behandelt hatte „mit großem Dank für zehn Jahre lebenswertes Leben“. Die schwere Krise, in der die Patientin sich seinerzeit sieben Jahre vor ihrem Tod befunden hatte, spielte offensichtlich in ihrer Erinnerung sieben Jahre später keine bedeutsame Rolle mehr. Wir können dies durchaus auch als Ausdruck einer erfolgreichen Rekonstruktionsarbeit werten. 



\section{Die Lebensgeschichte in der Denk- und Handlungstradition der Medizin}

Bei jedem Gespräch, das Ärzte/Ärztinnen mit ihren Patienten führen, erfahren diese Details aus dem Leben ihrer Patienten. Sie sind Ansprechpartner in einer Situation, in der ratsuchende Menschen sich sehr abhängig und hilfsbedürftig fühlen. Dies fördert meist das Bedürfnis von Patienten, aus ihrem Leben zu berichten und zwischen den Zeilen vermittelt sich dabei oft auch die Angst vor einem Verlust des bisherigen Lebenskontextes.

Ärztinnen und Ärzte werden hier Teilnehmer am Leben des Patienten eine sehr alte Funktion, die indirekt bereits im Eid des Hippokrates erwähnt wird. Insoweit sind Ärzte immer auch mit der Biographie ihrer Patienten befasst. Das trifft insbesondere dann zu, wenn Krankheit zur Krise führt und sich dann Krankengeschichte und Lebensgeschichte in biographischen Knotenpunkten verknüpfen.

Wie kann nun dies im alltäglichen ärztlichen Tun berücksichtigt werden?

Wahrscheinlich nur mit großen Schwierigkeiten! Denn unter den scharf kalkulierten Abläufen eines Großklinikums geht diese Leistung ärztlichen und auch pflegerischen - Handelns nicht in den Leistungskatalog ein. Das System der Fallpauschalen (DRG) belohnt eher die ,glatten“ Verläufe. Und selbst wenn neben der körperlichen Erkrankung eine weitere Erkrankung aus dem Bereich unseres Fachgebietes oder der Psychiatrie vorliegt, verbessert die zusätzliche Behandlung nicht die unmittelbare Erlössituation - allenfalls langfristig die Akzeptanz der klinischen Einrichtungen durch Patienten. 
Dies darf nicht so bleiben. Administrative Vorgaben und ökonomische Zwänge dürfen den ärztlichen Beruf - aber auch die Tätigkeit aller an der Behandlung Beteiligten - nicht auf die technische Durchführung von Behandlungsprozeduren reduzieren. Ich möchte an dieser Stelle aber davor warnen, die Verantwortlichen für diese Situation nur in der Politik zu suchen und unsere eigenen Probleme zu externalisieren; denn diese hängen auch wesentlich mit den Denk- und Handlungstraditionen der klinischen Medizin selbst zusammen:

Abb. 3

\section{Denk- und Handlungstradition der Medizin}

- Klinische Medizin als handlungsorientierte Wissenschaft

- Primäre Ausrichtung auf Diagnostik und Behandlung

- Wissenschaftliches Paradigma:

- die kontrollierte und randomisierte Studie

- mit statistisch objektivierbaren Befunden

- Objektive Befunde vs. subjektives Befinden

- Krankheit vs. Kranksein 
Von ihrem Selbstverständnis her ist die Medizin ja zunächst eine handlungsorientierte Wissenschaft und zwar zu Recht! Denn in Notfällen muss sie handlungsorientiert sein - mit einer primären Ausrichtung auf die Diagnostik und daraus abgeleiteten Interventionen. Das muss in vielen Situationen so sein und setzt sogar notweniger Weise beim Behandler eine gewisse Affekt-Isolierung voraus, um handlungsfähig zu bleiben. Diese im Bereich der Akutmedizin durchaus notwendige Denk- und Handlungstradition, ist aber bei der heute großen Zahl an chronischen Erkrankungen nicht ausreichend. Denn ohne Berücksichtigung der subjektiven Seite von Krankheit und Kranksein kann ein hinreichendes Verständnis für die bisherige Krankengeschichte und für zukünftige Verlaufsrisiken nicht gewonnen werden. Das gilt insbesondere, weil Krankheitsbewältigung (Coping) und Kooperation eines Patienten bei einer notwendigen Behandlung (Compliance) maßgeblich durch Biographie und prämorbide Persönlichkeit mitbestimmt sind. Und damit sind wir noch einmal bei meinem Thema, dass ich im Folgenden zusammenfassen möchte. 



\section{Die biographische Dimension von Krankheit und Kranksein und ihre Bedeutung in der Medizin}

Hanfried Helmchen (2007) hat vor kurzem noch einmal darauf hingewiesen: Krankheit und Kranksein müssen im jeweils lebensweltlichen Kontext der Patienten berücksichtigt werden, d. h. wir müssen die aktuelle Lebenssituation kennen, die sich aber nur vor dem Hintergrund einer Biographie für uns voll erschließt.

Abb. 4

Die biographische Dimension von Krankheit und Kranksein und ihre Bedeutung in der Medizin

- Krankheit und Kranksein im lebensweltlichen Kontext (Helmchen 2007)

- Krankheit als Bedrohung der Autonomie des Menschen

- Die grundsätzliche Unvollendbarkeit und Gebrochenheit menschlicher Existenz

(Jaspers 1965)

- Krankheit als tragischer Schicksalseinbruch (Dührssen 1998)

- Verlust des bisherigen und Wiedergewinnung eines neuen Lebens-Kontextes bei schwerer Krankheit 
Krankheit ist immer auch Bedrohung; sie gefährdet unsere Autonomie und Eigenständigkeit. Die entsprechenden Ängste werden aber von Patienten auf unterschiedliche Weise abgewehrt, oft ihrem Arzt nicht mitgeteilt und bleiben häufig hinter einem pseudoautonomen Verhalten verborgen.

Dabei tritt uns bei jeder schweren Erkrankung die grundsätzliche „Unvollendbarkeit und Gebrochenheit" menschlicher Existenz entgegen (Karl Jaspers 1965). Krankheit kann einen bis dahin gültigen Lebensentwurf in tragischer Weise zerstören. Dieses ,tragische Element in der menschlichen Existenz" muss nicht nur im Menschbild der Medizin, sondern nach Annemarie Dührssen (1998) auch in den anthropologischen Konzepten von Psychotherapieverfahren berücksichtigt werden, um eine allzu verflachende Sicht des menschlichen Seelenlebens zu verhindern.

\section{Verlust des bisherigen und Wiedergewinnung eines neuen Lebens-Kontextes bei schwerer Krankheit}

Oft geben uns Patienten aber nicht ohne Weiteres Informationen darüber, was sich in ihnen bei einem Verlust ihres bisherigen Lebenskontextes, bei einer schweren Erkrankung abspielt. Hier können uns wortmächtige Schriftsteller helfen, für die ihre eigene Erkrankung Anlass zum Schreiben geworden ist. Beispielhaft sei hier die Schriftstellerin Christa Wolf genannt (bekannt durch ihr frühes Werk „Der geteilte Himmel“). In ihrer 2002 publizierten Erzählung „Leibhaftig“ erfahren wir - plastischer als es manche Patienten beschreiben können, in denen aber dieselben Prozesse stattfinden! - viel über den kontextuellen Umbau einer Biographie - ausgelöst durch eine schwere lebensbedrohliche Erkrankung, die die Heldin der Erzählung einer existentiellen Krise aussetzt, die sie in der Endzeit der DDR in einem Zwischenreich von Leben und Tod erleidet.

In rezidivierenden septischen Fieberzuständen beschäftigt sie sich mit ihrer Beziehung zu den Menschen, die ihr Leben begleitet haben. In ihrem Be- 
richt wechselt sie zwischen der Ich-Form und dem beschreibenden „Sie“, wodurch wir mit in ihre Derealisations-und Depersonalisationszustände hineingezogen werden.

Auf dem Weg der Genesung erfährt sie vom Suizid einer ihr lange Zeit nahestehenden Person - man könnte sagen vom Tod ihres Alter Ego einem Repräsentanten ihres früheren Lebenskontextes. Am Ende verlässt die Patientin die Welt der Klinik als ein anderer Mensch.

In diesem schwierigen Prozess standen ihr Ärzte und Krankenschwestern - das schwesterliche spielte hier eine besondere Rolle! - zur Seite. Diese erhalten in dem regressiven, leicht paranoid getönten Zustand der Patientin deutlich überakzentuierte Zuschreibungen, die nicht unbedingt objektiv gerecht gewesen sein dürften. Auch das muss ein Behandlungsteam aushalten! Und von den entsprechenden Belastungen wissen viele kaum etwas, die nicht in der Krankenversorgung tätig sind. Dabei sind die Beteiligten Begleiter bei der Suche nach einem neuen Lebenskontext - ohne nach der „biographischen Wahrheit“"18 zu suchen. Es geht hier auch nicht um Psychotherapie im engeren Sinne - vielmehr um ein offenes Ohr, um Begleitung.

Bei diesem Prozess kommt offensichtlich eine bemerkenswerte Fähigkeit unseres autobiographischen Gedächtnisses zur Wirkung: So wie dieses uns ermöglicht, auf der Grundlage unserer bisherigen Welterfahrung Zukunft planvoll zu phantasieren - ohne dass diese immer zur Realität wird! - so scheint das autobiographische Gedächtnis auch retrospektiv auf der Grundlage einer geänderten Gegenwartserfahrung unser Bild von unserer eigenen Vergangenheit verändern zu können. Ein solcher Prozess findet in jedem Leben statt, insbesondere aber bei Brüchen im Lebensverlauf - z. B.

18 Zur Frage der „biographischen Wahrheit“ (vgl. S. Freud 1936; Küchenhoff 1996). 
bei schwerer Krankheit! - aber auch bei gesellschaftlichen und politischen Umwälzungen. ${ }^{19}$

${ }^{19}$ Vgl. vgl. hierzu Günter de Bruyn: Über Wahrheit und Dichtung in der Autobiographie 1995 


\section{Schlussbemerkung}

Unser subjektives Bild von unserer Biographie bestimmt maßgeblich das Bild von uns selbst. Nur unsere Biographie macht uns unverwechselbar und bestimmt letztlich unsere Identität. Eric Kandel, Psychiater und Neurowissenschaftler - verhinderter Psychoanalytiker - und Nobelpreisträger für Medizin und Physiologie hat es hier vor einiger Zeit bei einem Vortrag in Göttingen so ausgedrückt: „Wir sind Erinnerung“. Letztendlich ist es unser autobiographisches Gedächtnis, das uns von anderen Lebewesen unterscheidet - mit den Möglichkeiten, die Vergangenheit zu erinnern, die Zukunft zu antizipieren und uns in der Gegenwart mit anderen darüber auszutauschen.

Ich wünsche uns allen, dass diese conditio bumana auch in einer technisierten Hochleistungsmedizin erhalten bleibt.

Lassen Sie mich schließen mit einem Zitat von Gabriel García Márquez:

Nicht was wir gelebt haben, ist das Leben, sondern das, was wir erinnern

und wie wir es erinnern, um davon zu eræählen. ${ }^{20}$

20 In verdichteter Form hat Márquez die Beziehung zwischen historischer und narrativer Wahrheit glänzend eingefangen. In dem was wir anderen Menschen über unser 
Leben erzählen (Narrative) werden persönliche Ereignisse unseres Lebens zu einer subjektiven historischen Einheit konfiguriert und so unsere Existenz zu einem einheitlichen Ganzen gemacht. Damit tragen Narrative ganz wesentlich zu einer konturierten Ich-Identität bei. An dieser Stelle kann die diesbezügliche, umfassende Diskussion in Psychoanalyse und Sozialwissenschaften nicht nachgezeichnet werden. Bereits Sigmund Freud hatte sich zum Problem der „biographischen Wahrheit“ (1936) in einem Brief an Arnold Zweig geäußert. Eingehendere Erörterungen dieser Thematik aus psychoanalytischer Sicht finden sich bei Cremerius (1981), bei Roy Schafer (1981) und bei Küchenhoff (1996). Die sinnstiftende Funktion von Narrativen in ihrer Bedeutung für die Ich-Identität, aber auch für die kulturelle Entwicklung, hat der Kognitionspsychologe Jerome Bruner 1990 in einer ausgezeichneten interdisziplinären Studie dargestellt, die inzwischen auch in deutscher Übersetzung vorliegt (1997). Schließlich handelt der langjährige Direktor des Deutschen Historischen Instituts in Rom, Arnold Esch, in einem brillanten Essay „Zeitalter und Menschenalter" (1994) die Thematik aus der Sicht des Historikers ab - auch unter Rekurs auf den Göttinger Historiker Hermann Heimpel und den von ihm vor jetzt einem halben Jahrhundert geprägten Begriff vom „Menschen in seiner Gegenwart“ (1957). 


\section{Literatur}

Antonowsky, A (1987): Unravelling the Mystery of Health - How People Manage Stress and Stay Well. Jossey-Bass, San Francisco

Blankenburg, W. (1981): Nomotetische und idiographische Methodik in der Psychiatrie. Schweizer Archiv für Neurologie, Neurochirurgie und Psychiatrie, 128, 13-20

Blankenburg, W. (1989): Biographie und Krankheit. Georg-ThiemeVerlag, Stuttgart, New York

Boehme, G. (1990): Sinn und Gegensinn - über die Dekonstruktion von Geschichten. Psyche 44, 577-592

Boerner, P. (1964): Johann Wolfgang von Goethe. Rowohlt Reinbek/Hamburg

Bräutigam, W. (1989): Psychosomatik, Psychoanalyse, Psychotherapie - Alte und neue Herausforderung für die Medizin. Universitas 44, $762-772$

Bruner J. (1990): Acts of meaning. Harvard University Press, Cambridge MA. Deutsche Übersetzung: Sinn, Kultur und Ich-Identität. Carl-Auer, Heidelberg 1997

Bude, H. (1999): Lebenskonstruktionen als Gegenstand der Biographieforschung.

In: Jüttemann, G. u. Thomae, H. (Hrsg.): Biographische Methoden in den Humanwissenschaften. Belz, Weinheim und Basel, S. 247258

Cremerius, J. (1981): Die Konstruktion der biographischen Wirklichkeit im analytischen Prozeß. Freiburger literaturpsychologische Gespräche 1, 15-37

de Bruyn, G. (1995): Das erzählte Ich - Über Wahrheit und Dichtung in der Autobiographie. S. Fischer, Frankfurt/M

Dehli, M. (2007): Leben als Konflikt - Zur Biographie Alexander Mitscherlichs. Wallstein, Göttingen 
du Bois-Reymond, E. (1842): Zitiert nach Rüger 1996, S. 21. Vgl. auch Hoffmann et al. 1999, S. 3

Dührssen, A. (1981): Die Biographische Anamnese. Vandenhoeck und Ruprecht, Göttingen

Dührssen, A. (1998): Wesentliche anthropologische Konzepte in der psychoanalytisch orientierten Psychotherapie. Zsch. psychosom. Med. 44, 304-310

Esch, A. (1994): Zeitalter und Menschenalter - Der Historiker und die Erfahrung vergangener Gegenwart. C. H. Beck, München

Esser, G., Dinter R., Jörg M., Rose F., Villalba P., Laucht M., Schmidt M. H. (1993): Bedeutung und Determinanten der frühen MutterKind-Beziehung. Zsch. psychosom. Med. 39, 246-264

Franz M., Lieberz K., Schepank H. (2000): Seelische Gesundheit und neurotisches Elend. Der Langzeitverlauf in der Bevölkerung. Springer, Wien, New York

Freud S. (1895): Studien über Hysterie. GW. I, S. 75-312

Freud, S. (1936): Brief an Arnold Zweig vom 31.05.1936

Freyberger H.J., Freyberger H. (2007): Sechzig Jahre danach: Posttraumatische Belastungsstörungen, salutogene Faktoren und gutachterliche Einschätzungen bei Holocaust-Überlebenden im Langzeitverlauf. Z Psychosom Med Psychother 53, 380-392

Goethe, J. W (1812): Dichtung und Wahrheit 2. Teil (II, 6-10). Deutscher Klassiker Verlag Band 15, Frankfurt/M, 2007

Heimpel, H. (1957): Der Mensch in seiner Gegenwart. Vandenhoeck \& Ruprecht, Göttingen, zweite erweiterte Auflage.

Helmchen, H. (2007): Tragweite und Grenzen der Naturalisierung des Krankheitsbegriffes: Das Beispiel Psychiatrie. Vortrag vor der Guardini-Stiftung in Berlin, 20.02.2007

Hoffmann, S. O. (2006): Viktor von Weizsäcker - Arzt und Denker gegen den Strom - eine Würdigung des „Vaters der Psychosomatischen Medizin“ anlässlich des Erscheinens der Gesammelten Schriften. Deutsches Ärzteblatt 103, 577-578

Hoffmann, S. O., Liedtke, R., Schneider, W., Senf, W. (1999): Psychosomatische Medizin und Psychotherapie - Denkschrift zur Lage des Faches an den Hochschulen der Bundesrepublik Deutschland. Schatthauer, Stuttgart, New York 
Jaspers K. (1965): Allgemeine Psychopathologie. 8. Auflage, Springer, Berlin, Heidelberg, New York

Jones E. (1960): Das Leben und Werk von Sigmund Freud. Hans Huber, Bern, Stuttgart.

Kandel, E. (2006): Auf der Suche nach dem Gedächtnis. Siedler, München

Kierkegaard, S.: Verdichtet zitiert bei A. Esch (1994), S. 7. Originaltext siehe: Søren Kierkegaards Papirer. Bind 4A 164 S. 61; 2. erw. Aufl. hg. Von Nils Thulstrup, Gyldendal 1968

Küchenhoff, J. (1996): Zum Stellenwert der Biographie in der Psychoanalyse. Zsch. psychosom. Med. 42, 1-24

Lang, H. (2000): Das Gespräch als Therapie. Suhrkamp, Frankfurt/M

Markowitsch, H. u. Welzer, H. (2005): Das autobiographische Gedächtnis. Klett-Cotta, Stuttgart

Márquez, G. G. (2002): Leben, um davon zu erzählen. Kiepenheuer und Witsch, Köln 2002

Mitscherlich, A. (1954): Zur psychoanalytischen Auffassung psychosomatischer Krankheitsentstehung. Psyche 7, 561-578

Orlinsky, D. (2008): Die nächsten 10 Jahre Psychotherapie-Forschung - eine Kritik des herrschenden Forschungsparadigmas mit Korrekturvorschlägen. Psychother Psych Med 58, 345-354

Rudolf, G. (1990): Psychosomatik als Forschung und Therapie im Felde menschlicher Beziehungen. Zsch. psychosom. Med. 36, 276292

Rudolf, G. (1993): Aufbau und Funktion von Fallgeschichten im Wandel der Zeit. In: U. Stuhr, F. W. Deneke (Hrsg.): Die Fallgeschichte. Asanger, Heidelberg, S. 17-31

Rüger, U. (1996): Psychosomatische Medizin - Anmerkungen zur historischen Entwicklung und gegenwärtigen Situation. Georgia Augusta 64, 21-31

Rüger, U. (2007): Vierzig Jahre Richtlinien-Psychotherapie in Deutschland. Psychotherapeut 52, 102-111 
Rüger, U., Haase J., Fassl, K. (1996): Was Psychotherapeuten vom Leben ihrer Patienten (nicht) wissen. Zsch. psychosom. Med. 42, 329-342

Schafer, R. (1981): Narration in the Psychoanalytic Dialogue. In W. J. T. Mitchell (ed.): On Narrative. University of Chicago Press 1981, S. 31

Schepank, H., Franz M., Lieberz K., Häfner S. (2000): Gesundheit und psychische Erkrankungen im Verlauf von drei Jahrzehnten. Zusammenfassung. In: Franz M., Lieberz K., Schepank H. (Hrsg.) Seelische Gesundheit und neurotisches Elend. Der Langzeitverlauf in der Bevölkerung. Springer, Wien, New York, 149-157

Selye, H. (1955): Einführung in die Lehre vom Adaptationssyndrom. Georg-Thieme-Verlag, Stuttgart

Simmel, G. (1918): Lebensanschauung - Vier metaphysische Kapitel Viertes Kapitel: Das individuelle Gesetz. Duncker u. Humblot, München und Leipzig

Thomae, H. (1952): Die biographische Methode in den anthropologischen Wissenschaften. Studium Generale 5, 163-177

Tress, W (1986): „Das Rätsel der seelischen Gesundheit“. Vandenhoeck und Ruprecht, Göttingen

Vaillant, G. E. (1977): Adaption to life. Little Brown and Company, Boston, Toronto

Weizsäcker, V. von (1955): Gesammelte Schriften Band VII, S. 380 (Hrsg.: P. Achilles, D. Janz, M. Schrenk, C. F. von Weizsäcker) Suhrkamp, Frankfurt/M 1987

Werner, E (1989): „Sozialisation: Die Kinder von Kauai“. Spektrum der Wissenschaft, 118-123

Windelband W. (1894): Geschichte und Naturwissenschaft. Rektoratsrede, Straßburg

Wolf, Ch. (2002): Leibhaftig. Luchterhand, München 
Prof. Dr. med. Ulrich Rüger, 1986 bis 2007 Direktor der Abteilung

Psychosomatische Medizin und Psychotherapie im Zentrum Psychosoziale Medizin

der Medizinischen Fakultät

Georg-August-Universität Göttingen

urueger@gwdg.de 

Bei vielen schweren und chronischen Erkrankungen droht ein bis dahin tragfähiges Lebensgleichgewicht verloren zu gehen. Wie in keinem anderen Beruf sind daher Ärztinnen und Ärzte mit Krisen im menschlichen Leben konfrontiert und begleiten ihre Patienten oft dabei, einen neuen Lebenskontext zu gewinnen. Ulrich Rüger, langjähriger Lehrstuhlinhaber für Psychosomatische Medizin und Psychotherapie an der Georg-August-Universität Göttingen zeichnet in seiner Abschiedsvorlesung die enge Verbindung von Krankengeschichte und Lebensgeschichte nach und leistet damit einen Beitrag zur häufig vernachlässigten biographischen Dimension im Menschenbild der Medizin.

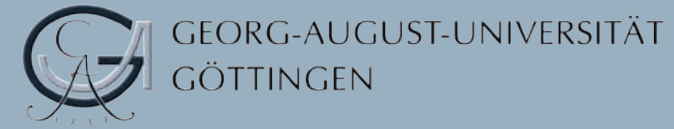

